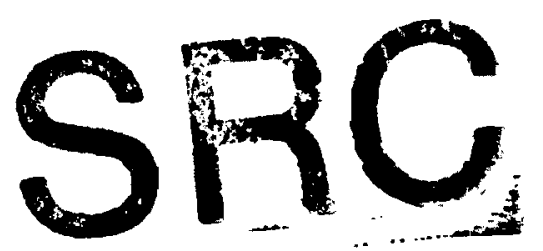

STABILITY OF COASTING BEAMS IN THE DEBUNCHER

J. MARRINER

$2 / 7 / 86$ 
Pbar Note 452 J. Marriner February 7, 1986

\section{STABILITY OF COASTING BEAMS IN THE DEBUNCHER}

The stability of high intensity beams has been a matter of some concern. While anti-proton operation uses beam currents of 10 a or less, it is sometimes useful to have proton beams with currents of the order of 1 ma and narrow (booster sized) momentum spreads. This note describes calculations of what can be expected for coasting beam instabilities and, in particular, the effect of the (high impedance) $53 \mathrm{MHz}$ cavities. This note specifically does not describe transverse instabilities, bunched beam instabilities, or turbulence in the debunching process.

The stability limit can be calculated using standard formulae (van der Meer CERN/PS/AA/80-4 and many others). The result of this calculation is the "stability plot" shown in figure 1a. This calculation assumes a 1 ma beam with a $\Delta \mathrm{p}(\sigma)$ of $2 \mathrm{MeV} / \mathrm{c}$. The curve (allowed impedance) scales inversely with beam current and $\Delta \mathrm{p}^{2}$. The momentum spread was assumed to be gaussian. Figures $1 \mathrm{~b}$ and $I c$ are the same plot but with different scales. The interpretation of this plot is that if the impedance of any device (or devices) is outside this curve, then the assumed beam distribution is unstable.

More rigorously one can make a so-called "Nyquist plot" where one plots $\mathrm{Z}_{\mathrm{w}}(w) / \mathrm{Z}_{\mathrm{b}}(w)$ where $\mathrm{Z}_{\mathrm{w}}$ is some wall impedance and $\mathrm{Z}_{\mathrm{b}}$ is the beam response (or beam impedance) shown in figure 1. These Nyquist plots are shown in figures 2abcd for different cavity tunes. Figure 2a shows the Nyquist plot for the 1 ma of beam assumed above assuming only 1 cavity which is tuned to resonance. The cavity has a shunt impedance of $1.8 \mathrm{Mn}$ and $a$ of 10,000 . The assumed beam distribution is unstable if the curve circles the point $(1,0)$ - quite close in figure 2a. Figure $2 b$ is the same except that 6 cavities are assumed. In figure $2 c$ the 6 cavities are tuned $100 \mathrm{kHz}$ below the beam revolution frequency (capacitive). In figure $2 \mathrm{~d}$ they are tuned $100 \mathrm{kHz}$ above (inductive). The inductive tuning is preferred, but either case should be stable. 
Conclusion:

The standard theory of the stability of coasting beams indicates that the high impedance $53^{\circ} \mathrm{MHz}$ Debuncher cavities do not upset the stability of moderate beam currents ( 1 ma) and narrow momentum spreads ( $\sigma=2$ Mev) provided that they are detuned. 


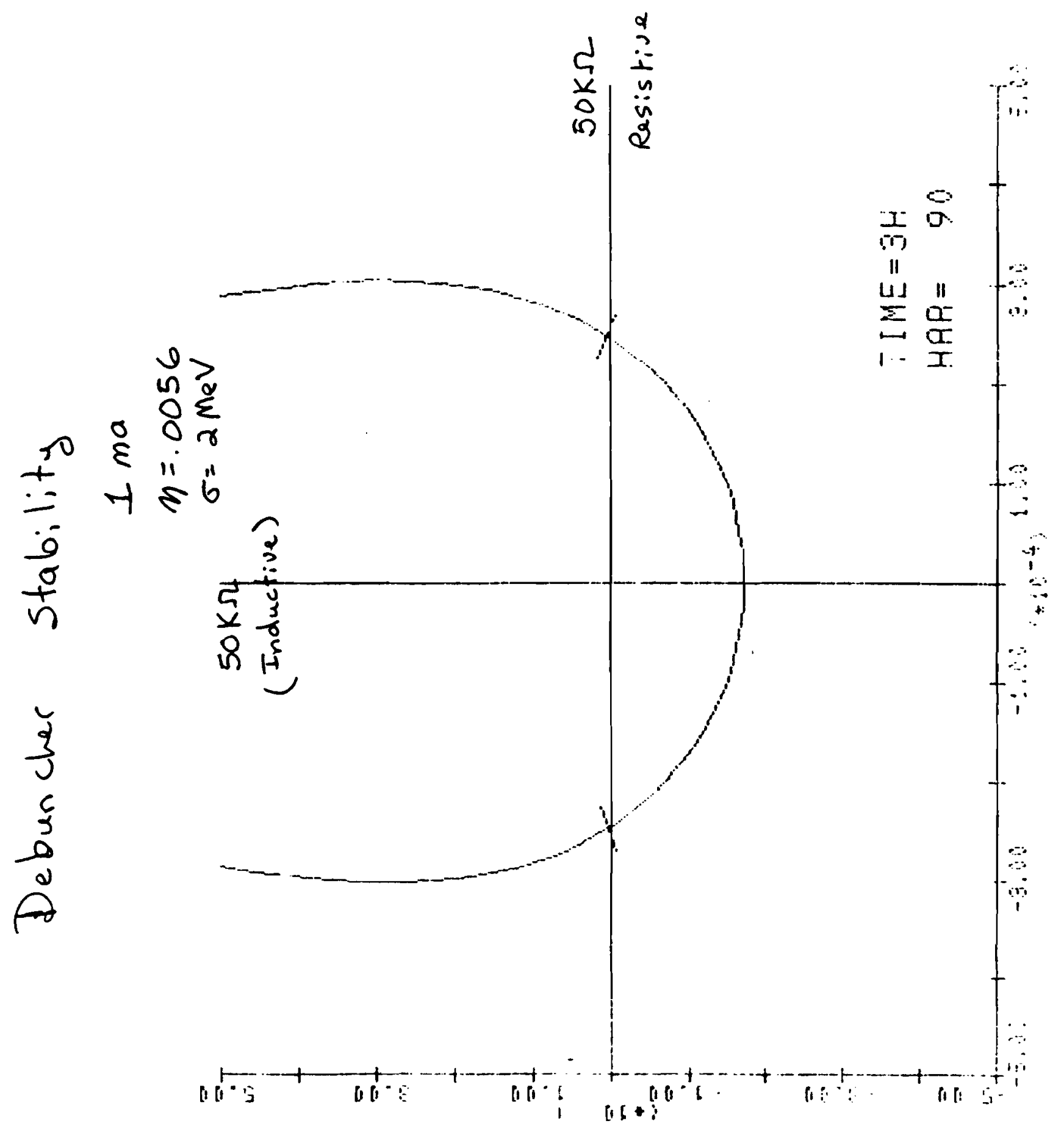




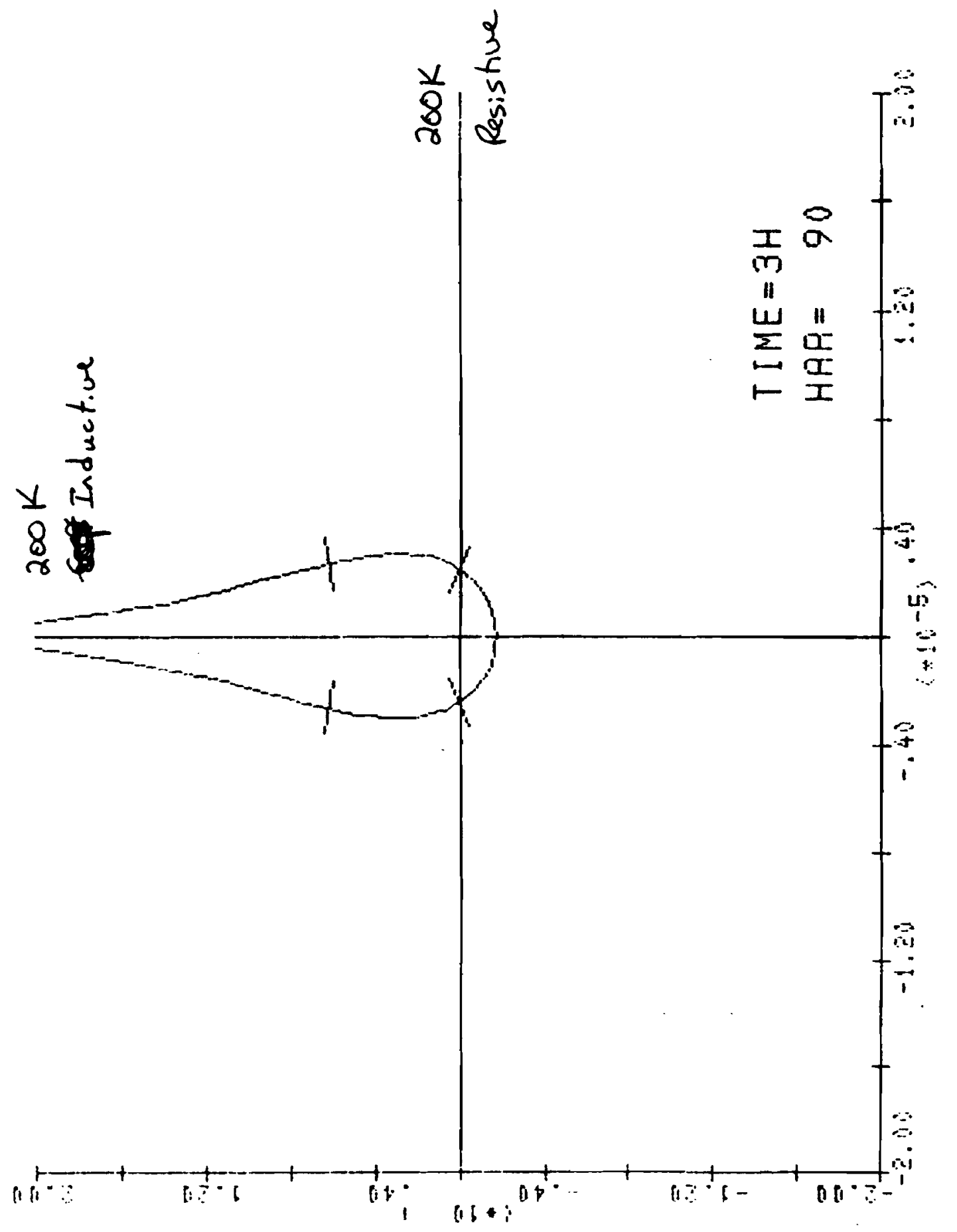




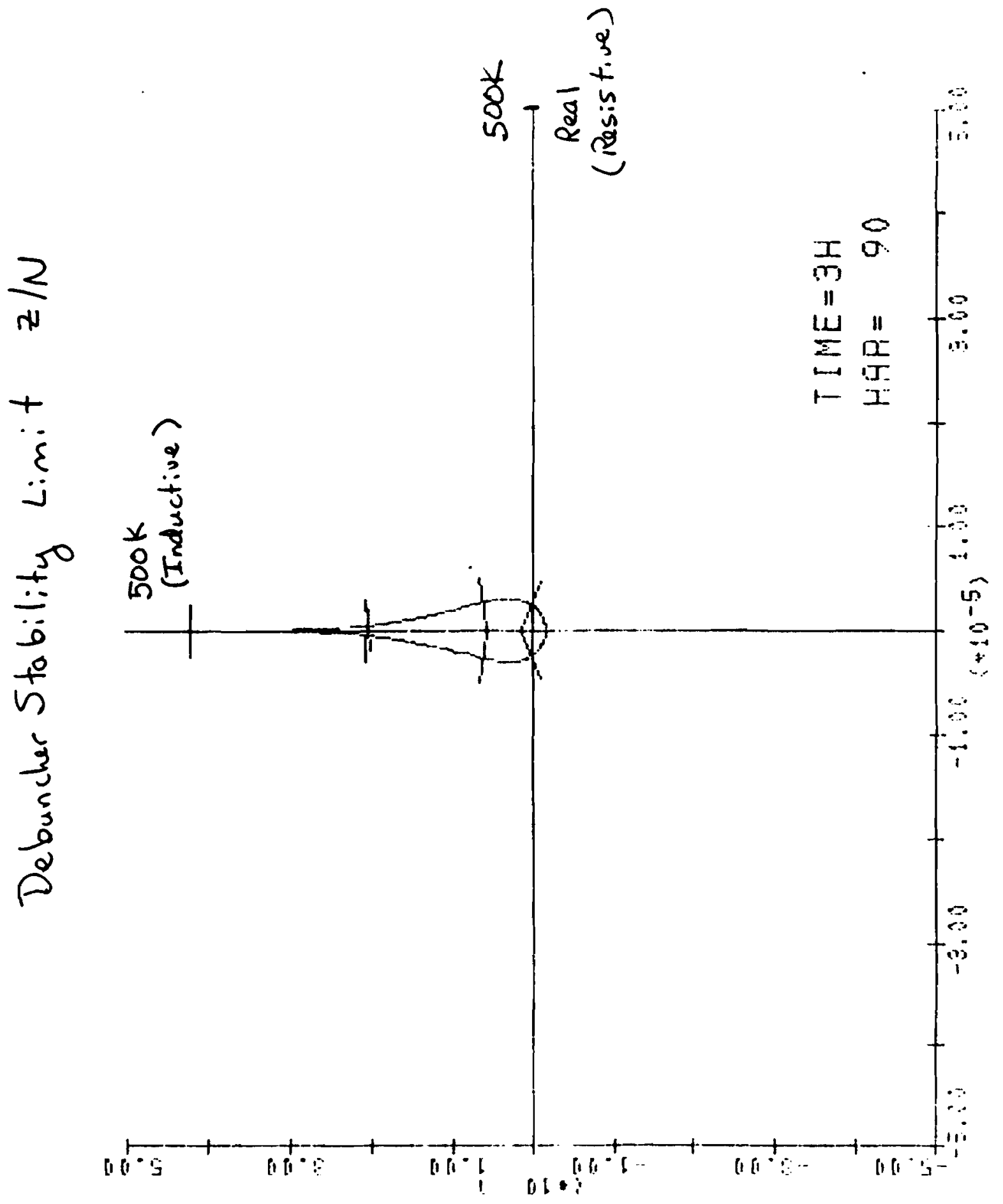









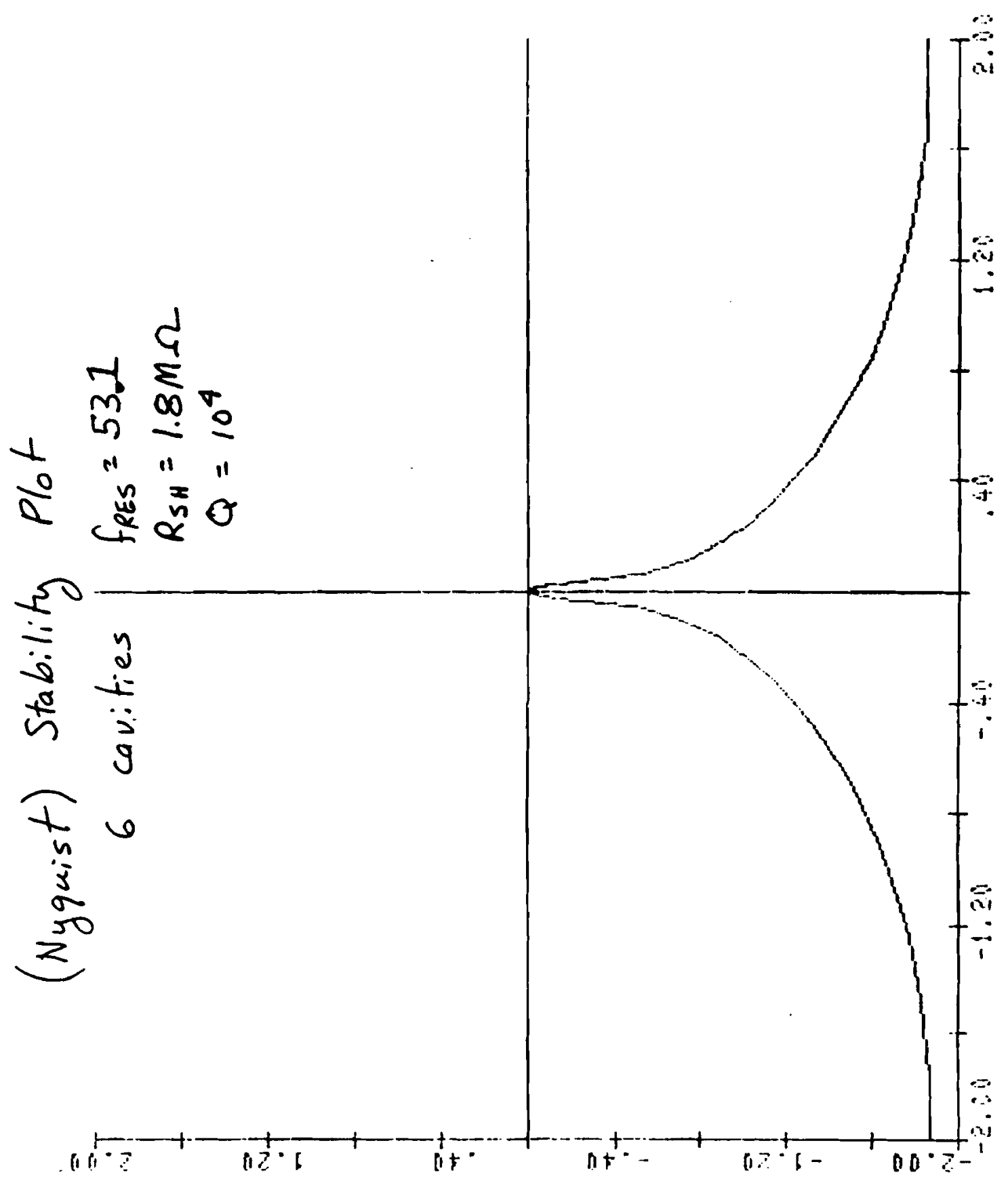









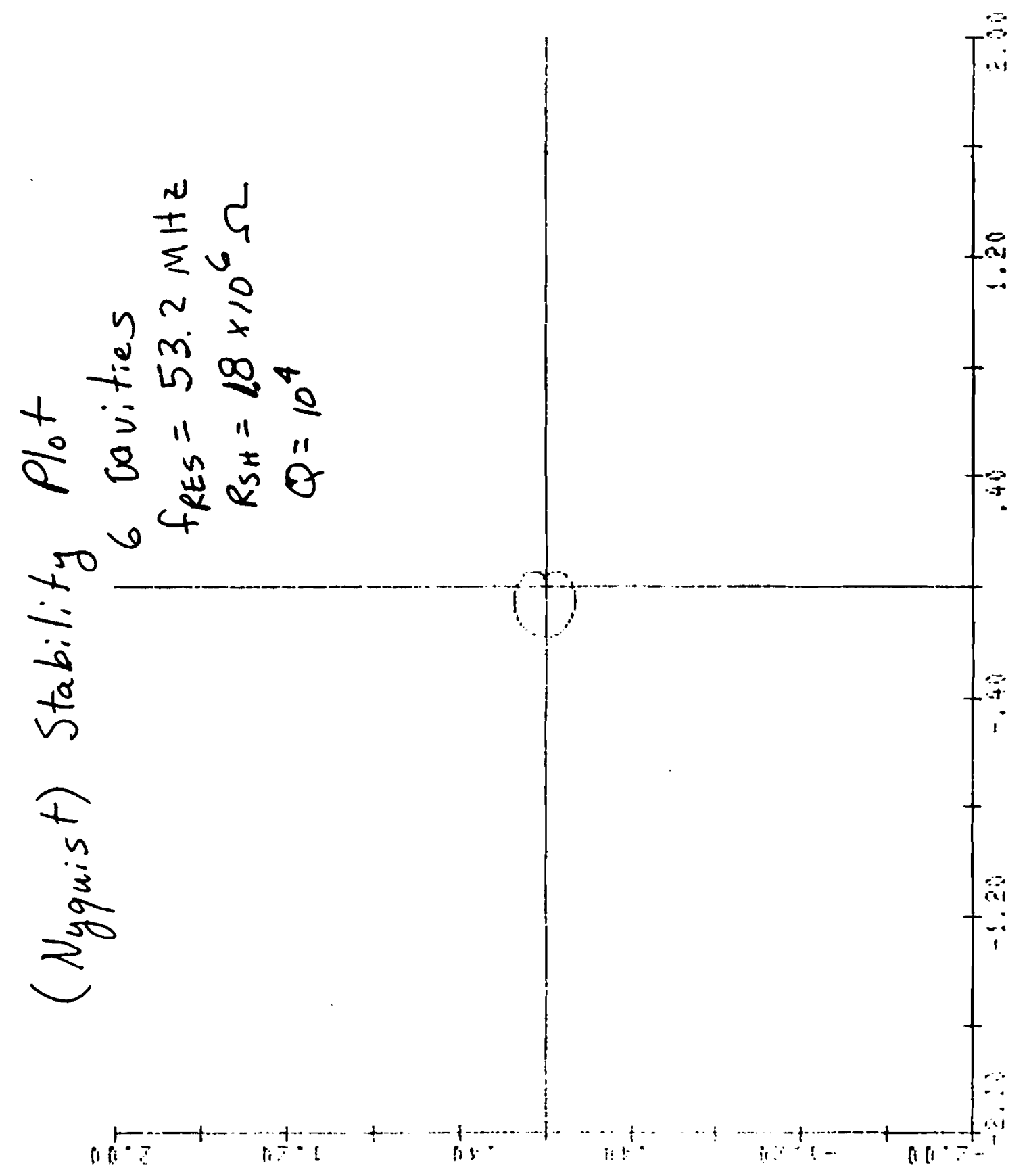

